\title{
Post-clear corneal phacoemulsification endophthalmitis: profile and management outcomes at a tertiary eye care center in western India
}

Aditya S. Kelkar ${ }^{1 *}$, Jai A. Kelkar ${ }^{1}$, Prajakta M. Barve ${ }^{1}$, Aishwarya Mulay ${ }^{1}$, Shubhangi Sharma ${ }^{1}$ and Winfried Amoaku ${ }^{2}$

\begin{abstract}
Background: Infectious endophthalmitis is a serious sight threatening intraocular inflammation that results from exogenous or endogenous spread of organisms into the eye.A retrospective case series to study the profile of endophthalmitis following clear corneal phacoemulsification in western India between years 2008 and 2014 was held in the National Institute of Ophthalmology, Pune, India. Cases of endophthalmitis post-clear corneal phacoemulsification were reviewed pertaining to demography, clinical history, surgeon experience, surgical complications, time of onset following surgery, duration between onset of symptoms and presentation to the center, presenting visual acuity and at follow-ups, slit-lamp examination and ultrasound findings, vitreous tap culture results, treatment, and final functional and anatomical outcomes.
\end{abstract}

Results: Of 60 cases, 34 were operated in the tertiary center and 26 were referred. The incidence of endophthalmitis post clear corneal phacoemulsification performed at the tertiary center was $0.17 \%$. Mean time delay between onset of symptoms and presentation to the tertiary care center was 2.6 days. Fifty percent cases were culture +ve, of which $80 \%$ were Gram +ve and $20 \%$ were Gram -ve, no fungal isolates. Coagulase -ve staphylococcus was the most common causative organism; rare isolates included Sphingomonas paucimobilis and Streptococcus mitis. Twenty-six eyes underwent primary vitrectomy. Mean presenting visual acuity was $2.14 \pm 0.07$ logMAR units which improved to logMAR $0.98 \pm 0.12$ at final follow-up. Presenting VA was $>20 / 200$ in $13.3 \%$ and $<$ HM in $60 \%$ cases. $66.7 \%$ of eyes had visual improvement; $26.7 \%$ cases achieved VA $20 / 40$ at final follow-up. Gram +ve and culture -ve cases, better presenting VA, and less time delay between onset and presentation had a favorable visual outcome.

Conclusions: The shift of the clinico-microbiological spectrum of endophthalmitis could be due to change in surgical technique to clear corneal phacoemulsification. Predictors of good visual outcome include good presenting visual acuity, early presentation to the center, culture negativity, and coagulase negative organisms.

Keywords: Endophthalmitis, Microbiology, Presenting visual acuity, Time of presentation, Visual outcome

\footnotetext{
*Correspondence: adityapune4@gmail.com

${ }^{1}$ National Institute of Ophthalmology, 1187/30 Off Ghole Road, Near

Mahatma Phule Museum, Shivajinagar, Pune 411005, India

Full list of author information is available at the end of the article
} 


\section{Background}

Postoperative endophthalmitis is an intraocular inflammatory condition due to infection from microbial organisms (bacteria, fungi or, on rare occasions, parasites) that enter the eye during the perioperative period [1]. Endophthalmitis is potentially the most devastating complication of cataract surgery, and despite optimal management, the visual outcome is poor in many cases [2]. Early diagnosis and aggressive treatment with appropriate antimicrobial therapy, as well as surgical intervention, are mandatory for optimal visual outcomes. In India, cataract surgery continues to be the most common cause of postoperative endophthalmitis [3-6].

Several risk factors have been described as associated with post-cataract endophthalmitis, including the type of surgery, incision site, seniority of surgeon, surgical complications, and systemic factors [5-7]. A few studies have previously reported on post-cataract endophthalmitis from different parts of India as well as abroad [2-13]. These reports suggest that there may be differences in occurrence/incidence, type of surgery associated with higher predisposition, and the causative infective organisms in the different geographic areas previously studied. However, to the best of our knowledge, there are no studies from western India on the subject.

The purpose of this study was to investigate the profile of endophthalmitis following clear corneal phacoemulsification at a tertiary eye care center in western India, with emphasis on the clinical presentation, risk factors, microbiological profile, treatment outcomes, and predictors of good visual outcome.

\section{Methods}

This was a retrospective study of all eyes that were diagnosed as having endophthalmitis following clear corneal phacoemulsification through superior or temporal incisions between January 2008 and December 2014, and with at least 3 months follow-up in a tertiary eye care center in western India. Data included patients operated at the tertiary eye care center, as well as referred patients who were operated elsewhere within the region. All cases included in the study were operated by a fully trained ophthalmologist. Data was collected by reviewing the endophthalmitis register and the electronic medical records of all cataract surgeries operated between January 2008 and December 2014. Patients who developed endophthalmitis following small incision cataract surgery (SICS), phacoemulsification through scleral tunnel, and phacoemulsification combined with filtration surgery/vitrectomy and patients with inadequate data regarding the type of cataract surgery were excluded from the analysis. This study received approval from our institutional ethics committee and complied with the tenets of the Declaration of Helsinki.
Clinical diagnosis of endophthalmitis was based on the presence of a combination of one or more of presence of hypopyon, fibrinous membrane in anterior chamber, and vitreous haze. The presence of other symptoms and signs, such as pain, photophobia, reduced vision, ciliary injection, eyelid, or corneal edema, was supportive of the diagnosis.

All patients with endophthalmitis were admitted in the first instance. The treatment was guided by Early Vitrectomy Study (EVS) [14] protocol. Vitrectomy was done immediately followed by intravitreal injection of antibiotics (vancomycin $1.0 \mathrm{mg} / 0.1 \mathrm{~mL}$ and ceftazidime $2.25 \mathrm{mg} / 0.1 \mathrm{~mL}$ ) and steroids (dexamethasone $4 \mathrm{mg} /$ $0.1 \mathrm{~mL}$ ) only if patients presented with vision worse than hand motions (IVAS + PPV group). When the presenting vision was hand motions or better, an initial vitreous tap/biopsy followed by intravitreal antibiotics (vancomycin $1.0 \mathrm{mg} / 0.1 \mathrm{~mL}$ and ceftazidime $2.25 \mathrm{mg} / 0.1 \mathrm{~mL}$ ) and steroids (dexamethasone $4 \mathrm{mg} / 0.1 \mathrm{~mL}$ ) was performed (IVAS group). These were repeated as clinically indicated, by non-response, usually at $48 \mathrm{~h}$. Systemic antibiotics were not administered. The procedures were carried out in the operation theater under full aseptic precautions.

Samples for microbiologic evaluation were collected from the aqueous humor or vitreous humor just before intravitreal administration of antibiotics or during vitrectomy and were sent to the laboratory for microscopy for Gram staining, potassium hydroxide mount, culture, and sensitivity. One hourly topical fortified vancomycin (5\%), ceftazidime (5\%), prednisolone eye drops (1\%), and tropicamide eye drops $(0.8 \%)$ at bedtime were started and tapered gradually at 3-day intervals depending on the response to treatment. No systemic antibiotics were given. A favorable anatomical outcome was defined as the absence of any inflammation at the end of the treatment period. A favorable functional outcome was deemed if the eye achieved best-corrected visual acuity (VA) $>20$ / 200 at the final follow-up visit.

Data collected included patient demography, clinical history (including posterior capsule rupture), details of surgery including surgeon grade/seniority, surgical complications, time of onset following cataract surgery, duration between onset of symptoms and presentation to the eye care center, symptoms and signs, presenting visual acuity, and at follow-up examinations after endophthalmitis (1 week, 1 month, 3 month, 6 month, and last follow-up), slit-lamp examination findings, ultrasound $B$ scan findings, aqueous or vitreous tap for culture and sensitivity, type of treatment, and final functional and anatomical outcomes.

Statistical analysis was performed using the statistical software SPSS, version 20.0 (Chicago, IL, USA). The association of final visual acuity with various risk factors 
was analyzed by using chi-square test or Fisher's exact test (expected cell frequency $<5$ ). Subgroup analysis of intravitreal steroids with vitrectomy group (IVAS + PPV) vs. intravitreal injection and steroids group (IVAS) was done using Student's unpaired $t$ test. Comparison between mean final visual outcomes with respect to culture positivity was done using ANOVA test. A $p$ value $<0.05$ was considered statistically significant.

\section{Results}

The total number of phacoemulsification surgeries performed in the tertiary center over the study period was 19,541. All these were clear corneal superior site phacoemulsification procedures, and $403(2.06 \%)$ had posterior capsular tear (PC tear). We were unable to determine the total number of phacoemulsification procedures undertaken in each of the referral centers.

A total of 60 patients of endophthalmitis following clear corneal phacoemulsification were evaluated for the study of which 26 patients were referred from other centers. Of these, four patients (6.66\%), all from the tertiary eye care center, had eventful phacoemulsification with posterior capsular rupture. In all four cases, anterior vitrectomy was performed and the IOL was placed in the sulcus. Other cases of endophthalmitis occurred in two eyes following SICS, two following phacoemulsification through scleral tunnel, and three eyes where there was inadequate data regarding the type of cataract surgery performed and were excluded from the analysis. Amongst the 60 cases, a superior corneal incision was used in $48(80 \%)$ and a temporal corneal incision in 12 (20\%) cases. All eyes with endophthalmitis following temporal incision phacoemulsification were referred from other centers. Excluding referred cases, the incidence of endophthalmitis following clear corneal phacoemulsification surgeries at our tertiary center was $0.17 \%$. The incidence of endophthalmitis in cases complicated by a posterior capsular rent was $0.99 \%$. The annual incidence of endophthalmitis in the tertiary center following clear corneal phacoemulsification is summarized in Table 1 . The incidence of endophthalmitis for the referred cases due could not be calculated due to lack of adequate data. None of the referred cases of endophthalmitis were complicated by posterior capsular tear.

All the cases including referred patients were operated by fully trained ophthalmologists. The seniority of the operating surgeon is summarized in Table 2 and Additional file 1: Table S1 and shows that the junior surgeons had a higher occurrence of endophthalmitis for the patients operated in the tertiary center, although this difference was not statistically significant ( $p$ value $=0.38$ by chi-square test). We were unable to determine such figures for the referred cases due to
Table 1 Incidence of endophthalmitis 2008-2014

\begin{tabular}{llc}
\hline Year & $\begin{array}{l}\text { No. of cases of endophthalmitis/total } \\
\text { no. of cases operated at the tertiary } \\
\text { eye care center }\end{array}$ & Incidence \\
\hline 2008 & $6 / 2062$ & 0.29 \\
2009 & $6 / 2386$ & 0.25 \\
2010 & $4 / 2611$ & 0.15 \\
2011 & $5 / 2791$ & 0.18 \\
2012 & $6 / 3568$ & 0.17 \\
2013 & $4 / 2988$ & 0.13 \\
2014 & $3 / 3135$ & 0.09 \\
Total & $34 / 19,541$ & 0.17 \\
\hline
\end{tabular}

lack of adequate data. The mean duration of followup was 183 days (90-930 days).

\section{Demographic data}

There were $32(53.3 \%)$ males and 28 (46.7\%) were females. The mean age of our patients was 72.3 years (range 58-85 years). Endophthalmitis was always unilateral and occurred in the right eye in $36(60 \%)$ and the left eye in 24 (40\%) of cases. Associated systemic risk factors included diabetes mellitus in 8 (13.3\%), systemic hypertension in 14 (23.3\%), diabetes and hypertension in 4 (6.7\%), and others including bronchial asthma and ischemic heart disease in $4(6.7 \%)$ of cases. No systemic risk factors were identified in $30(50 \%)$ of cases.

\section{Presenting visual acuity}

The mean presenting VA was $2.14 \pm 0.07 \log$ MAR units. Presenting VA was $>20 / 200(<1.0 \log$ MAR $)$ in $8(13.33 \%)$ and $20 / 200$ to $>\mathrm{HM}+(\log M A R \quad 1.0-2.2)$ in $16(26.7 \%)$. A subset of 36 cases $(60 \%)$ presented with visual acuity of $\leq$ hand movements (HM) (logMAR > 2.3), including 10 (16.7\%) with HM (logMAR 2.3$)$, and 26 (43.3\%) who had VA of $<$ HM $(\operatorname{logMAR}>2.3)$ and therefore proceeded to vitrectomy.

\section{Clinical presentation}

The time interval from surgery to presentation with endophthalmitis varied from 1 day to 3 months. The majority of the patients $34(56.66 \%)$ presented within the

Table 2 Seniority of surgeons - tertiary center operated cases

\begin{tabular}{lll}
\hline $\begin{array}{l}\text { Surgical experience } \\
\text { of the surgeon in } \\
\text { years }\end{array}$ & $\begin{array}{l}\text { No of cases of endophthalmitis } \\
\text { at tertiary eye care center/no } \\
\text { of cases operated }\end{array}$ & $\begin{array}{l}\text { Incidence of } \\
\text { endophthalmitis }\end{array}$ \\
\hline$>10$ years & $7 / 5180$ & $0.13 \%$ \\
$5-10$ years & $17 / 10,397$ & $0.16 \%$ \\
$1-5$ years & $10 / 3964$ & $0.25 \%$ \\
Total & $34 / 19,541$ & $0.17 \%$ \\
\hline
\end{tabular}


first postoperative week, median -7 days (range 190 days) (Table 3 ).

The mean time delay between onset of symptoms, as determined from the history, and presentation to the eye care center was 2.6 days (range $2 \mathrm{~h}-15$ days).

Treatment was initiated within $2 \mathrm{~h}$ of presentation to the eye care center in all cases. This time interval between onset of symptoms and presentation to the eye care center was significant and affected the final visual outcome ( $p$ value $<0.001$ ), with cases presenting earlier having better final visual outcome (Fisher's exact test). (see Additional file 1: Table S2).

While all 60 patients $(100 \%)$ presented with a chief complaint of hazy vision, $36(60 \%)$ patients had associated complaints such as pain, redness, watering, and floaters, whereas rest $24(40 \%)$ did not have any associated complaints. Of the 60 cases, $36(60 \%)$ had hypopyon, and all $60(100 \%)$ eyes had vitreous haze clinically which was confirmed on B scan ultrasonography at presentation. Ciliary injection was observed in 28 (47.7\%), eyelid edema in 18 (30.0\%), corneal haze in 34 (56.7\%), and fibrinous pupillary membrane in $20(33.3 \%)$ of cases.

\section{Microbiology}

The analyses of aqueous and vitreous samples revealed positive bacterial etiology in 30 cases (50\%). Fungi were not detected in any of the samples. Of the positive isolates, $24(80 \%)$ cases were Gram-positive and 6 (20\%) were Gram-negative bacteria. Coagulase negative staphylococcus was the most common causative organism. Amongst the Gram-negative cases, Escherichia coli was the most commonly identified. These microbiology results are summarized in Table 4 .

Streptococcus mitis was identified in one eye with endophthalmitis of late onset (6 weeks postoperatively). The eye with Sphingomonas paucimobilis endophthalmitis presented on the first day postoperatively. Both cases improved to $20 / 200$ at the final follow-up visit.

\section{Treatment}

Intravitreal antibiotics and steroid (IVAS) was given in all patients as described previously. Of the 32 patients in the IVAS group, 24 required only one injection, 6 cases

Table 3 Interval from surgery to presentation with endophthalmitis

\begin{tabular}{lll}
\hline Time interval & No. of cases & \% of cases \\
\hline $1-7$ days & 34 & 56.66 \\
$8-14$ days & 6 & 10.00 \\
$15-28$ days & 10 & 16.66 \\
$1-2$ months & 8 & 13.33 \\
$2-3$ months & 2 & 3.33 \\
Total & 60 & 100 \\
\hline
\end{tabular}

required two injections with an interval of $48 \mathrm{~h}$ between two consecutive injections, and 2 cases required three injections for non-resolving inflammation; in 1 case, this next injection was administered $48 \mathrm{~h}$ after the second injection, and in the other case, it was administered 1 week following the second injection.

A cohort of 28 patients required pars plana vitrectomy (PPV) with intravitreal antibiotics (IVAS + PPV group), 26 patients underwent primary 25 gauge pars plana core vitrectomy along with intravitreal antibiotic and steroid injection, while in two (3.3\%) cases, vitrectomy was done after three intravitreal injections due to non-resolving inflammation.

Amongst the 26 cases that underwent primary vitrectomy with injection, 10 required one more intravitreal injection and 8 required two more injections, with an interval of $48 \mathrm{~h}$ between two consecutive injections.

\section{Final visual acuity and correlations}

The mean VA at final follow-up was logMAR $0.98 \pm$ 0.12. A favorable functional outcome (VA $<\log M A R 1.0$ or $20 / 200)$ was seen in 40 (66.7\%) eyes, with $26.7 \%$ cases achieving VA better than $0.3 \log$ MAR $(20 / 40)$ at final follow-up. Table 5 shows the correlation between presenting and final visual acuities. All eyes with VA $>\mathrm{HM}$ improved following treatment, with a final VA better than $20 / 200$ in $83.33 \%$ of cases. In the 36 eyes that presented with VA of $<\mathrm{HM}(\log \mathrm{MAR}>2.3)$, BCVA improved in $77.8 \%$ of cases, with $20 / 200$ achieved in only $55.6 \%$ of cases.

Eyes with good presenting VA ( $\geq 20 / 200)$ had a $71.5 \%$ improvement in VA with a mean final visual outcome of $\log$ MAR $0.29 \pm 0.11$, while those with presenting VA of $<20 / 200$ (mean $\log$ MAR $2.32 \pm 0.05$ ) had a final VA of $\log$ MAR $1.09 \pm 0.13$ (Table 6).

The difference between the mean presenting VA and final visual outcome was statistically significant $(p$ value $=$ 0.001 ). A total of 20 patients had $\mathrm{VA}<20 / 200$ at final follow-up.

The microbial culture-negative cases showed better mean VA at presentation $(\log$ MAR $2.03 \pm 0.09)$. Their posttreatment outcomes were also better, with a mean

Table 4 Microbiology-culture results

\begin{tabular}{ll}
\hline Gram-positive organisms & $\begin{array}{l}\text { Gram-negative organisms } \\
6(20 \%)\end{array}$ \\
\hline Coagulase negative Staphylococcus .8 & E. coli 3 \\
MRSA coagulase negative 5 & Pseudomonas 1 \\
Staphylococcus aureus 3 & Klebsiella 1 \\
Streptococcus pneumoniae 3 & Sphingomonas paucimobilis 1 \\
Propionibacterium acnes 1 & \\
Staphylococcus epidermidis 3 & \\
Streptococcus mitis 1 &
\end{tabular}


Table 5 Correlation between presenting and final VA

\begin{tabular}{llll}
\hline Presenting VA subgroups & No. of cases & $\begin{array}{l}\text { No. of cases with final VA better than } \\
\text { presenting VA }\end{array}$ & $\begin{array}{l}\text { No. of cases with final VA < logMAR 1 } \\
(>20 / 200)\end{array}$ \\
\hline$\geq 20 / 200(\leq 1$ logMAR) & 8 & $8(100.0 \%)$ & $8(100.0 \%)$ \\
$<20 / 200-H M+($ logMAR 1.1-logMAR 2.3) & 16 & $16(100.0 \%)$ & $12(75.0 \%)$ \\
$\leq$ HM $(\geq \log$ MAR 2.3) & 36 & $28(77.8 \%)$ & $20(55.6 \%)$ \\
\hline
\end{tabular}

final VA of $\operatorname{logMAR} 0.89 \pm 0.16$. The mean final visual outcome was better than 20/200 in culture-negative and Gram-positive subgroups while the mean final visual outcome was worse than 20/200 in Gram-negative subgroup. Figure 1 shows the inter-group comparison of presenting vision and final vision $\log$ Mar based on microbiology. Overall, $66.7 \%$ of Gram-positive cases and $73.3 \%$ of culture-negative cases improved to $20 / 200$ or better while only $33.33 \%$ of Gram-negative cases improved to $20 / 200$ or better. Thus, Gram-positive and culturenegative cases seemed to have a favorable visual outcome while eyes with Gram-negative cultures had a worse final visual outcome. This difference was, however, not statistically significant ( $p$ value $=0.18$ by ANOVA test). These are summarized in Additional file 1: Table S3.

The mean final VA was slightly better in the IVAS group (logMAR $0.89+0.15)$ versus combination of PPV + IVAS (logMAR $1.09+0.18)$ although the difference was not statistically significant ( $p$ value $=0.38$, Student's unpaired $t$ test) (Table 7).

By using multivariate binary logistic regression with response variable as the final outcome of vision and with risk factors as age, time delay between onset of symptoms and presentation; day of presentation; presenting features such as corneal haze, hypopyon, flare, and cells; vitreous haze; and pupillary membrane, there is a significant association with time interval $>1$ day ( $p$ value $=0.046$, odds ratio of 4.794 with $95 \% \mathrm{CI}$ ) with poor visual outcome.

Causes of poor visual outcome included retinal detachment (three eyes), non-resolving vitreous haze (three), pigment dispersion over the lens (four), epiretinal membrane (four), macular hole (one), corneal decompensation (four), and macular infarction with sclerosed vessels and optic atrophy (one). The one diagnosed with macular hole 2 months post-vitrectomy for endophthalmitis subsequently underwent vitrectomy with ILM peeling and gas tamponade. At final follow-up, this eye achieved a BCVA of 20/125 (logMAR 0.8) with closure of macular hole. Three eyes had rhegmatogenous retinal detachment following vitrectomy for endophthalmitis. Two of these eyes underwent vitrectomy with silicone oil but had a poor final visual outcome, in spite of favorable anatomical attachment of the retina. One eye had PVR with funnel retinal detachment and had no further intervention.

\section{Discussion}

Endophthalmitis has been studied in great detail in the past, with various publications from western countries $[9,11,12]$ and China [15] as well as the Indian subcontinent $[3-6,13]$. The previous studies from India were mainly during the era of extracapsular cataract extraction (ECCE), and over the transition from ECCE to phacoemulsification, and were from the north and southern parts of the country. This study, to the best of our knowledge, represents an attempt to fill the void in the literature from western India on endophthalmitis following clear cornea incision phacoemulsification.

This study aimed at evaluating the profile of all cases of post-phacoemulsification endophthalmitis patients with emphasis on the clinical presentation, microbiological profile, treatment outcomes, and predictors of good visual outcome. The catchment area of this study was predominantly urban, located in the western part of India.

The reported incidence of post-cataract surgery endophthalmitis has varied, ranging from $<0.05$ to $>0.3 \%$ [11, 12, 16-19]. The incidence of endophthalmitis after clear corneal cataract surgery in this study of $0.17 \%$ is comparable to that from other previous studies. Similarly, posterior capsule rupture shows the higher association with endophthalmitis reported in previous studies $[5,11,12]$. In our study, the male predilection and the age range of 48 to 85 years (mean, 72 years) are similar to that reported in other studies from elsewhere $[13,14,20,21]$. Similarly, the occurrence of hypopyon as a presenting feature in $60 \%$ and corneal involvement in $10 \%$ of our cases are comparable to $50-85.7 \%$ of hypopyon $[1,13,14,20,22]$ and $4.8-9 \%$

Table 6 Correlation between presenting VA, final VA, and visual improvement across presenting VA subgroups

\begin{tabular}{|c|c|c|c|}
\hline Presenting visual acuity subgroup & Mean presenting VA (logMar) & Mean final VA (logMar) & Improvement (\%) \\
\hline $\mathrm{VA} \geq 20 / 200(\leq \log M A R \quad 1)(n=8)$ & $1.00 \pm 0.00$ & $0.29 \pm 0.11$ & 71.5 \\
\hline VA $<20 / 200(>\log M A R$ 1) $(n=52)$ & $2.32 \pm 0.05$ & $1.09 \pm 0.13$ & 55.4 \\
\hline
\end{tabular}

Values are mean \pm standard error of mean (SEM) 


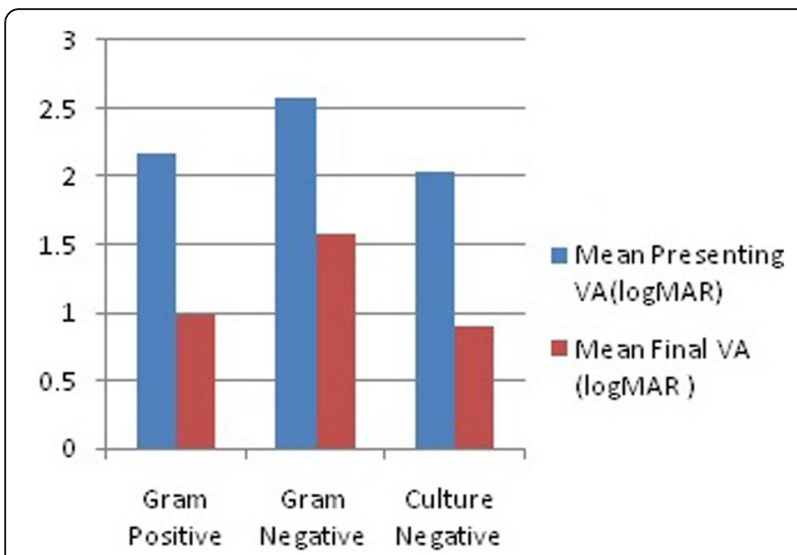

Fig. 1 The inter-group comparison of presenting vision and final vision logMar: based on microbiology

of corneal infiltrate previously reported elsewhere [13, 14]. Gupta et al. [13] and Kamalarajah et al. [2] had reported a presenting VA $<20 / 200$ in the majority (92.75 and $85 \%$, respectively) of the patients which is similar to our study (86.66\%).

The EVS study [14] has reported a $69.3 \%$ culture-positive rate from biopsies/taps in post-phacoemulsification endophthalmitis. In the various Indian studies, culture positivity of endophthalmitis cases was reported to be $38-58 \%$ $[4,13,20]$. The culture-positive rate of $50 \%$ from our study is comparable to these earlier reported figures. The microbiological results from our study revealed coagulase negative Staphylococci as the most common causative organism in concordance with the world literature on endophthalmitis [23] and some Indian studies [3, 20]. However, a study of post-cataract endophthalmitis from north India [13] which included 70.5\% of ECCE surgeries showed a marked microbiological variation, with $57.5 \%$ fungal cases, and Aspergillus flavus as the most common organism. Another study from south India [6] reported Nocardia species as the most common isolated organism, accounting for more than half of their relatively small sample size. This difference could be attributed to variations in the sample size, type of surgery, and geographical/environmental variation. Additional file 1: Table S4 gives a comparison between culture-positive rates in this study in comparison to a few other studies.

An interesting finding in our study is the identification of rare causative organisms such as $S$. mitis and S. paucimobilis.
S. mitis acute endophthalmitis has previously been reported post-intravitreal injections and after other intraocular surgeries with devastating visual outcomes in majority of patients; however, this organism is not usually associated with post-cataract surgery endophthalmitis with late presentation [24, 25]. S. paucimobilis, an aerobic, Gramnegative bacillus, is another rarely reported causative organism in post-cataract surgery.

Various Indian studies have reported final visual outcomes $>20 / 200$ in $33 \%$ [13] to $54.8 \%$ [20] cases. In our study, the final VA was $>20 / 200$ in $40(66.7 \%)$ cases. This positive difference may be explained by the earlier presentation and prompt institution of management using the EVS protocol. The difference in the causative organisms may also partly explain the better outcomes than previously reported from north and south India where fungal endophthalmitis was a more common cause of endophthalmitis $[13,20]$. One of the important predictors of final visual outcome is the presenting visual acuity [21, 22]. Similar to studies reported by Gower et al. [22] and Lalwani et al. [21], the results from the present study observed that a better presenting visual acuity resulted in better final visual outcome.

Good visual outcome in our study could be attributed to prompt initiation of treatment within $2 \mathrm{~h}$ after diagnosis of endophthalmitis and a predominance of coagulase negative staphylococci and culture-negative cases which are generally associated with a less severe disease and hence better visual outcome [21, 26, 27]. Similar to the EVS study [14], predisposing factors for poor visual outcome in our study were poor presenting visual acuity and type of organism on culture.

Retinal/choroidal detachment, corneal edema, epiretinal membrane, vitreous opacities, neovascular glaucoma, and phthisis bulbi have previously been reported as the major causes of poor visual outcome in postcataract endophthalmitis [21]. The causes of poor visual outcome in the present study are similar to those previously described, except that none of the eyes (in our study) developed phthisis bulbi.

Although the data was meticulously collected in this study, the retrospective non-comparative design of study, lack of polymerase chain reaction (PCR) testing in all patients are obvious limitations. However, we believe that this report from a large tertiary referral unit, which included data gathered over the course of 6 years,

Table 7 Treatment outcomes—comparison between IVAS + PPV vs. IVAS

\begin{tabular}{|c|c|c|c|c|}
\hline Parameters & Injection + vitrectomy group (IVAS + PPV) $(n=28)$ & Injection group (IVAS) $(n=32)$ & $T$ value & $P$ value \\
\hline Presenting vision (logMAR) & $2.19 \pm 0.11$ & $2.11 \pm 0.09$ & 0.540 & $0.591^{N S}$ \\
\hline Final vision (logMAR) & $1.09 \pm 0.18$ & $0.89 \pm 0.15$ & 0.890 & $0.377^{\mathrm{NS}}$ \\
\hline$\%$ Improvement & $52.6 \%$ & $61.9 \%$ & -1.082 & $0.284^{\mathrm{NS}}$ \\
\hline
\end{tabular}

Values are mean \pm standard error of mean (SEM). $P$ values by Student's unpaired $t$ test. $P$ value $<0.05$ is considered to be statistically significant NS statistically non-significant 
reflects the current scenario of endophthalmitis in western India. Polymerase chain reaction (PCR) accompanied with cloning and sequencing is one of the most sensitive, specific, and rapid molecular techniques in the detection of microbial species in clinical specimens like infectious endophthalmitis [28]. Fifty percent of patients in the current study were culture negative of which $26.7 \%$ had poor visual outcome. We feel that in these cases, PCR would have probably improved the chances of identification of organisms, which would have resulted in better outcomes in this group of patients.

\section{Conclusions}

Post-cataract surgery endophthalmitis has wide geographical variations. In the current study, the predictors of good visual outcome in post-phacoemulsification endophthalmitis include a good presenting visual acuity, less time delay between onset of symptoms and presentation to the eye care center, culture negativity, and coagulase negative Staphylococcus as the causative organism. Urbanization, availability of modern technology for early diagnosis, and prompt management of endophthalmitis and change in surgical technique from extracapsular cataract extraction to phacoemulsification could be the reason for a shift of the clinico-microbiological spectrum of endophthalmitis, ultimately resulting in better visual outcomes.

\section{Additional file}

Additional file 1: Table S1. Seniority of surgeon- referred cases. Table S2. Correlation between time interval between onset of symptoms and presentation to the tertiary eye care center and visual outcomes. Table S3. Microbiological organism vs. visual outcomes. Table S4. Comparison of culture positivity and microbiologic spectrum in different studies. (DOCX $20.3 \mathrm{~kb}$ )

\section{Abbreviations}

BCVA: Best-corrected visual acuity; Cl: Confidence interval; ECCE: Extracapsular cataract extraction; EVS: Endophthalmitis vitrectomy study; IVAS: Intavitreal antibiotics and steroids; PC: Posterior capsular; PCR: Polymerase chain reaction; PPV: Pars plana vitrectomy; RR: Relative risk; SEM: Standard error of mean; SICS: Small incision cataract surgery; VA: Visual acuity

\section{Acknowledgements}

The authors thank Mr Shrivallabh Sane (statistician) for the statistical analysis of data.

\section{Funding}

None.

\section{Availability of data and materials}

The dataset(s) supporting the conclusions of this article is (are) included within the article (and its additional file).

\section{Authors' contributions}

$\mathrm{AK}, \mathrm{JK}, \mathrm{PB}, \mathrm{AM}$, and SS contributed to the conception and design, acquisition of data, analysis and interpretation of data, drafting of the manuscript or revising it critically for important intellectual content, and final approval of the version to be published. WA contributed to the concept and design, analysis and interpretation of data, drafting of the manuscript and revising it critically for important intellectual content, and final approval of the version to be published. All authors agree to be accountable for all aspects of the work.

\section{Competing interests}

The authors declare that they have no competing interests.

\section{Consent for publication}

Not applicable.

\section{Ethics approval and consent to participate}

This study received approval from our institutional ethics committee and complied with the tenets of the Declaration of Helsinki. Written informed consent was taken before undergoing surgical procedures/injections from the patients included in the study.

\section{Declarations}

No author has a financial or proprietary interest in any material or method mentioned.

\section{Author details}

${ }^{1}$ National Institute of Ophthalmology, 1187/30 Off Ghole Road, Near Mahatma Phule Museum, Shivajinagar, Pune 411005, India. ${ }^{2}$ Division of Ophthalmology and Visual Sciences, University of Nottingham, Nottingham NG7 2RD, UK.

Received: 31 August 2016 Accepted: 17 November 2016

Published online: 28 November 2016

\section{References}

1. Barry P, Seal DV, Gettinby G, for the ESCRS Endophthalmitis Study Group et al (2006) ESCRS study of prophylaxis of postoperative endophthalmitis after cataract surgery; preliminary report of principal results from a European multicenter study. J Cataract Refract Surg 32:407-410, erratum, 709

2. Kamalarajah S, Silvestri G, Sharma N et al (2004) Surveillance of endophthalmitis following cataract surgery in the UK. Eye Jun 18(6):580-587

3. Jambulingam M, Parameswaran SK, Lysa S et al (2010) A study on the incidence, microbiological analysis and investigations on the source of infection of postoperative infectious endophthalmitis in a tertiary care ophthalmic eye care center: an 8-year study. Indian J Ophthalmol 58(4):297-302. doi:10.4103/0301-4738.64132

4. Sharma S, Padhi TR, Basu S et al (2014) Endophthalmitis patients seen in a tertiary eye care centre in Odisha: a clinico-microbiological analysis. Indian J Med Res 139(1):91-98

5. Khanna RC, Ray VP, Latha M et al (2015) Risk factors for endophthalmitis following cataract surgery-our experience at a tertiary eye care centre in India. Int J Ophthalmol 8(6):1184-1189. doi:10.3980/j.issn.2222-3959.2015.06. 19. eCollection 2015

6. Lalitha P, Rajagopalan J, Prakash K et al (2005) Post cataract endophthalmitis in south India: incidence and outcome. Ophthalmology 112:1884-1889

7. Wong TY, Chee SP (2004) The epidemiology of acute endophthalmitis after cataract surgery in an Asian population. Ophthalmology 111(4):699-705

8. Koc F, Sen E, Demirbay P et al (2002) Factors influencing treatment results in pseudophakic endophthalmitis. Eur J Ophthalmol 12:34-39

9. Cooper BA, Holekamp NM, Bohigian G, Thompson PA (2003) Case-control study of endophthalmitis after cataract surgery comparing scleral tunnel and clear corneal wounds. Am J Ophthalmol 136:300-305

10. Nagaki Y, Hayasaka S, Kadoi C et al (2003) Bacterial endophthalmitis after small-incision cataract surgery: effect of incision placement and intraocular lens type. J Cataract Refract Surg 29:20-26

11. Colleaux KM, Hamilton WK (2000) Effect of prophylactic antibiotics and incision type on the incidence of endophthalmitis after cataract surgery. Can J Ophthalmol 35:373-378

12. Miller JJ, Scott IU, Flynn HW Jr et al (2005) Acute-onset endophthalmitis after cataract surgery (2000-04): incidence, clinical settings and visual acuity outcomes after treatment. Am J Ophthalmol 139:983-987

13. Gupta A, Gupta V, Gupta A et al (2003) Spectrum and clinical profile of post cataract surgery endophthalmitis in north India. Indian J Ophthalmol 51 139-145

14. Results of Endophthalmitis Vitrectomy Study (1995) A randomized trial of immediate vitrectomy and of intravenous antibiotics for the treatment of 
postoperative bacterial endophthalmitis. Endophthalmitis Vitrectomy Study Group. ArchOphthalmol 113:1479-1496

15. Ding Y, Lin M, Liu H et al (2011) Outcomes of post-cataract surgery endophthalmitis referred to a tertiary center from local hospitals in the south of China. Infection 39:451-460

16. Endophthalmitis Study Group, European Society of cataract and Refractive Surgeons (2007) Prophylaxis of postoperative endophthalmitis following cataract surgery: results of the ESCRS multicenter study and identification of risk factors. J Cataract Refract Surg 33:978-988

17. Ravindran RD, Venkatesh $R$, Chang DF et al (2009) Incidence of post-cataract endophthalmitis at Aravind Eye Eye care center. Outcomes of more than 42,000 consecutive cases using standardized sterilization and prophylaxis protocols. J Cataract Refract Surg 35(4):629-636. doi:10.1016/j.jcrs.2009.01.002

18. Jabbarvand M, Hashemian HKhodaparast M, Jouhari M et al (2016) Endophthalmitis occurring after cataract surgery. Outcomes of more than 480, 000 cataract surgeries, epidemiologic features, and risk factors. Ophthalmology 123:295-301. doi:10.1016/j.ophtha.2015.08.023

19. Creuzot-Garcher C, Benzenine E, Mariet AS, de Lazzer A et al (2016) Incidence of acute postoperative endophthalmitis after cataract surgery. A nationwide study in France from 2005 to 2014. Ophthalmology 123(7): 1414-1420. doi:10.1016/j.ophtha.2016.02.019

20. Das T, Hussain A, Naduvilath T et al (2012) Case control analyses of acute endophthalmitis after cataract surgery in South India associated with technique, patient care, and socioeconomic status. J Ophthalmol 2012: 298459. doi:10.1155/2012/298459

21. Lalwani GA, Flynn HW Jr, Scott IU et al (2008) Acute-onset endophthalmitis after clear corneal cataract surgery (1996-2005). Clinical features, causative organisms, and visual acuity outcomes. Ophthalmology 115(3):473-476

22. Gower EW, Keay LJ, Stare DE et al (2015) Characteristics of endophthalmitis after cataract surgery in the United States medicare population. Ophthalmology 122(8):1625-1632. doi:10.1016/j.ophtha.2015.04.036

23. Han DP, Wisniewski SR, Wilson LA et al (1996) Spectrum and susceptibilities of microbiologic isolates in the Endophthalmitis Vitrectomy Study. Am J Ophthalmol 122:1-17

24. Chen E, Ho AC, Garg SJ et al (2009) Streptococcus mitis endophthalmitis presenting as frosted branch angiitis after intravitreal pegaptanib sodium injection. Ophthalmic Surg Lasers Imaging 40:192-194

25. Mao LK, Flynn HW Jr, Miller D, Pflugfelder SC (1992) Endophthalmitis caused by streptococcal species. Arch Ophthalmol 110:798-801

26. Combey de Lambert A, Campolmi N, Cornut PL, French Institutional Endophthalmitis Study Group et al (2013) Baseline factors predictive of visual prognosis in acute postoperative bacterial endophthalmitis in patients undergoing cataract surgery. JAMA Ophthalmol 131(9):1159-1166. doi:10.1001/jamaophthalmol.2013.4242

27. Okhravi N, Towler HM, Hykin P et al (1997) Assessment of a standard treatment protocol on visual outcome following presumed bacterial endophthalmitis. Br J Ophthalmol 81(9):719-725

28. Majid A, Behnam H, Mojtaba A et al (2015) PCR detection and identification of bacterial contaminants in ocular samples from post-operative endophthalmitis. J Clin Diagn Res 9(4):NC01-NC03. doi:10.7860/JCDR/2015/10291.5733

\section{Submit your manuscript to a SpringerOpen ${ }^{\circ}$ journal and benefit from:}

- Convenient online submission

- Rigorous peer review

- Immediate publication on acceptance

- Open access: articles freely available online

- High visibility within the field

Retaining the copyright to your article 